\title{
Statistical hyperbolicity of relatively hyperbolic groups
}

\author{
JEREMY OSBORNE \\ WEN-YUAN YANG
}

\begin{abstract}
We prove that a nonelementary relatively hyperbolic group is statistically hyperbolic with respect to every finite generating set. We also establish the statistical hyperbolicity for certain direct products of two groups, one of which is relatively hyperbolic.
\end{abstract}

20F65, 20F67

\section{Introduction}

The idea of statistical hyperbolicity was first introduced by M Duchin, S Lelièvre, and C Mooney in [7]. Let $G$ be a group generated by a finite set $S$. Assume that $1 \notin S=S^{-1}$. Denote by $\mathscr{G}(G, S)$ the Cayley graph of $G$ with respect to $S$. Consider the natural combinatorial metric on $\mathscr{G}(G, S)$, denoted by $d$, inducing a word metric on $G$. The intuitive meaning of statistical hyperbolicity of a group can then be summed up as follows: on average, random pairs of points $x, y$ on a sphere of the Cayley graph of the group almost always have the property that $d(x, y)$ is nearly equal to $d(x, 1)+d(1, y)$. More precisely,

Definition 1.1 Denote $S_{n}=\{g \in G \mid d(1, g)=n\}$ for $n \geq 0$. Define

$$
E(G, S)=\lim _{n \rightarrow \infty} \frac{1}{\left|S_{n}\right|^{2}} \sum_{x, y \in S_{n}} \frac{d(x, y)}{n}
$$

if the limit exists. The pair $(G, S)$ is called statistically hyperbolic if $E(G, S)=2$.

Recall that a group is called elementary if it is a finite group or a finite extension of $\mathbb{Z}$. It is easily checked that an elementary group is not statistically hyperbolic with respect to any generating set. In [7], Duchin, Lelièvre, and Mooney proved that $\mathbb{Z}^{d}$ for $d \geq 2$ is not statistically hyperbolic for any finite generating set. It was also discovered by Duchin and Mooney in [8] that the integer Heisenberg group with any finite generating set is not statistically hyperbolic.

A list of statistically hyperbolic examples were also found in [7]: 
Examples 1.2 (1) Nonelementary hyperbolic groups for any finite generating set.

(2) Direct product of a nonelementary hyperbolic group and a group for certain finite generating sets.

(3) The lamplighter groups $\mathbb{Z}_{m} \prec \mathbb{Z}$, where $m \geq 2$ for certain generating sets.

We remark that a definition of statistical hyperbolicity analogous to the above can be considered for any metric space with a measure. (For graphs we consider the counting measures). We refer the reader to [7] for precise definitions. For any $m, p \geq 2$, the Diestel-Leader graph $\operatorname{DL}(m, p)$ is proved to be statistically hyperbolic in [7]. In [5], Dowdall, Duchin, and Masur established statistical hyperbolicity for the Teichmüller space with various measures.

Summarizing the above results, one could think of the number $E(G, S)$ as a measurement of negative curvature in groups and spaces. So it would be natural to expect that the statistical hyperbolic property holds for a more general class of groups with negative curvature. A natural source of such groups to be investigated is the class of relatively hyperbolic groups, which generalizes word hyperbolic groups and includes many more examples, such as

(1) fundamental groups of nonuniform lattices with negative curvature (see Bowditch [1]),

(2) free products of groups, or a finite graph of groups with finite edge groups,

(3) limit groups (see Dahmani [4]), and

(4) $\mathrm{CAT}(0)$ groups with isolated flats (see Hruska and Kleiner [14]).

We refer the reader to Section 2 and references therein for more details on relatively hyperbolic groups. The purpose of this article is to generalize the first two items in Examples 1.2 to the setting of relatively hyperbolic groups.

Recently, Osborne established in his thesis [15] that relatively hyperbolic groups are statistically hyperbolic, provided that the group growth rate dominates those of parabolic subgroups. Our first result is to drop this assumption and to establish the full generalization of the aforementioned result of Duchin, Lelièvre and Mooney in relatively hyperbolic groups.

Theorem 1.3 A nonelementary relatively hyperbolic group is statistically hyperbolic with respect to every finite generating set. 
Let's say a bit about the ingredients in proof of our theorem. It was observed in [7] that statistical hyperbolicity appears to be more delicate than the usual metric notion of hyperbolicity in the sense of Gromov. Namely, examples of trees can be produced to have arbitrary number $E(G, S) \in[0,2]$. These examples do not have many isometries because they are not homogeneous. Thus in their proof of statistical hyperbolicity for hyperbolic groups, Duchin, Lelièvre, and Mooney make essential use of a result of Coorneart [3] about growth functions. This is recently generalized by Yang in [17] for relatively hyperbolic groups; see Lemma 2.9. Apart from this, we also exploit a crucial fact in [17] to obtain the full generality: parabolic groups have convergent Poincaré series; see Corollary 2.8. Based on them, our proof roughly follows the outline in the hyperbolic case but with more involved analysis.

We now state our second result about a direct product of two groups, one of which is relatively hyperbolic. First recall the notion of the growth rate $v_{G, S}$ of a group $G$ relative to $S$, which is defined to be the limit

$$
v_{G, S}=\lim _{n \rightarrow \infty} \frac{\log \left|S_{n}\right|}{n} .
$$

A generating set $S$ for $G \times H$ is called split if every generator in $S$ lies either in $G$ or in $H$. Denote $S_{G}:=S \cap G$ and $S_{H}:=S \cap H$. Taking into account Theorem 1.3, we obtain the following theorem extending a similar result in [7].

Theorem 1.4 Let $G \times H$ be a direct product of a nonelementary relatively hyperbolic group $G$ and a group $H$. Let $S$ be a split finite generating set for $G \times H$, with $S_{G}$ and $S_{H}$ the corresponding generating sets for $G$ and $H$. If $v_{G, S_{G}}>v_{H, S_{H}}$, then $(G \times H, S)$ is statistically hyperbolic.

It is obvious that Theorem 1.4 can be thought of as a generalization of Theorem 1.3.

Lastly, we derive a corollary of Theorem 1.4. Recall that a group has subexponential growth if its growth rate is zero for some (thus any) generating set. It is well-known that a nonelementary relatively hyperbolic group has exponential growth.

Corollary 1.5 A direct product of a nonelementary relatively hyperbolic group and a group of subexponential growth is statistically hyperbolic with respect to finite split generating sets.

This article is structured as follows. Section 2 prepares preliminary material to be used in the proof of Theorem 1.3, which occupies the whole of Section 3. In Section 4, we give a proof of Theorem 1.4. 
Acknowledgements Osborne would like to thank Chris Hruska for directing the research of this topic in the context of his $\mathrm{Ph} \mathrm{D}$ dissertation. Thanks also to Moon Duchin and Chris Mooney for originally formulating the question which ultimately led to this paper. Yang is supported by the Chinese grant "The Recruitment Program of Global Experts". We are grateful to the referee for a careful reading and many useful comments and, in particular, for providing a simple argument to significantly shorten our proof of Lemma 3.5 .

\section{Preliminaries}

Consider the Cayley graph $\mathscr{G}(G, S)$ of $G$ with respect to $S$. Define

$$
B(1, n)=\{g \in G \mid d(1, g) \leq n\} .
$$

Let $S_{n}$ be the set of elements $g \in G$ such that $d(1, g)=n$. It will be useful to consider the spherical set in a subgroup $H$ in $G$. Define

$$
S_{n}(H)=H \cap S_{n} .
$$

A parametrized path $p$ from $p_{-}$to $p_{+}$is endowed with a natural order. For any two (parametrized) points $v, w \in p$, we denote by $[v, w]_{p}$ the segment between $v$ and $w$ in $p$. As usual, $[v, w]$ denotes a (choice of) geodesic between $v$ and $w$. Our path $p$ is often endowed with a length parametrization $p:[0, \ell(p)] \rightarrow \mathscr{G}(G, S)$.

Let $p$ and $q$ be two geodesics with the common initial endpoint $p_{-}=q_{-}$. A point $w \in q$ is called congruent relative to $v \in p$ if $d\left(v, p_{-}\right)=d\left(w, p_{-}\right)$.

Given a subset $X$ in $\mathscr{G}(G, S)$, the projection $\operatorname{Proj}_{X}(v)$ of a point $v$ to $X$ is the set of nearest points in $X$ to $v$. For a subset $A \subset \mathscr{G}(G, S)$, we define

$$
\operatorname{Proj}_{X}(A)=\bigcup_{a \in A} \operatorname{Proj}_{X}(a) .
$$

\section{A Relative hyperbolicity and contracting property}

Given a finite collection of subgroups $\mathcal{P}$ in $G$, one can talk about the relative hyperbolicity of $G$ with respect to $\mathcal{P}$. From various points of view, the notion of relative hyperbolicity has been considered by many authors: Gromov [12], Bowditch [2], Osin [16], Drutu and Sapir [6], and Gerasimov [9], just to name a few. These theories of relatively hyperbolic groups emphasize different aspects and are widely accepted to be equivalent for finitely generated groups. We refer the interested reader to Hruska [13] and Gerasimov and Potyagailo [10] for further discussions on their equivalence. 
In order to avoid heavy exposition, we collect here only necessary facts in the theory of relatively hyperbolic groups. Let $\mathbb{P}=\{g P \mid g \in G, P \in \mathcal{P}\}$. Then $\mathbb{P}$ plays an important role in the geometry of $\mathscr{G}(G, S)$, which has the following nice property.

Definition 2.1 Let $\epsilon, D>0$. A subset $X$ is called $(\epsilon, D)$-contracting in $\mathscr{G}(G, S)$ if

$$
\operatorname{Diam}\left(\operatorname{Proj}_{X}(\gamma)\right)<D
$$

for any geodesic $\gamma$ in $\mathscr{G}(G, S)$ with $N_{\epsilon}(X) \cap \gamma=\varnothing$.

A collection of $(\epsilon, D)$-contracting subsets is referred to as a $(\epsilon, D)$-contracting system. The constants $\epsilon, D$ will be often omitted if no confusion happens.

We now recall some useful properties of contracting sets and refer the reader to [18] for detailed discussions.

Lemma $2.2[6 ; 11 ; 18]$ Let $(G, \mathcal{P})$ be a relatively hyperbolic group. Then $\mathbb{P}$ is a contracting system with the following two equivalent properties.

(1) Bounded intersection property For any $\epsilon>0$ there exists $R=\mathcal{R}(\epsilon)>0$ with

$$
\operatorname{Diam}\left(N_{\epsilon}(X) \cap N_{\epsilon}\left(X^{\prime}\right)\right)<R
$$

for any two distinct $X, X^{\prime} \in \mathbb{P}$.

(2) Bounded projection property There exists a finite number $D>0$ such that

$$
\operatorname{Diam}\left(\operatorname{Proj}_{X}\left(X^{\prime}\right)\right)<D
$$

for any two distinct $X, X^{\prime} \in \mathbb{P}$.

Proof The contracting property was established in [11, Proposition 8.5]. Property (1) was proved in [6, Theorem 4.1] and in [11, Proposition 5.6], and property (2) was proved in [10, Proposition 3.27]. The equivalence was shown in [18, Lemma 2.3].

Hereafter, we will often invoke the function $\mathcal{R}$ without explicit mention of Lemma 2.2. The following notion was introduced by Hruska [13], and further elaborated on by Gerasimov and Potyagailo [11].

Definition 2.3 Fix $\epsilon, R>0$. Let $\gamma$ be a path in $\mathscr{G}(G, S)$ and $v \in \gamma$ a vertex. Given $X \in \mathbb{P}$, we say that $v$ is $(\epsilon, R)$-deep in $X$ if it holds that $\gamma \cap B(v, R) \subset N_{\epsilon}(X)$. If $v$ is not $(\epsilon, R)$-deep in any $X \in \mathbb{P}$, then $v$ is called an $(\epsilon, R)$-transition point of $\gamma$. 
In what follows, we assume that there exists a uniform constant $\epsilon_{0}>0$ such that Lemmas 2.4, 2.5 and 2.6 hold. The first lemma is a consequence of the contracting property of $\mathbb{P}$ (without the assumption of relative hyperbolicity). See [17, Lemma 2.9] for a proof.

Lemma 2.4 Let $p$ be a geodesic and a point $v \in p$ be $(\epsilon, R)$-deep in some $X \in \mathbb{P}$ for $\epsilon \geq \epsilon_{0}$ and $R=\mathcal{R}(\epsilon)$. Denote by $x$ and $y$ the entry and exit points of $p$ in $N_{\epsilon}(X)$, respectively. Then $x$ and $y$ are $\left(\epsilon_{0}, R\right)$-transition points.

The following lemma could be derived using techniques in [13, Section 8] or it follows from the proof of [10, Proposition 7.1.1] in terms of Floyd distance.

Lemma 2.5 Let $\epsilon \geq \epsilon_{0}$ and $R=\mathcal{R}\left(\epsilon_{0}\right)$. There exists $D=D(\epsilon, R)$ with the following property. Consider a geodesic triangle consisting of three geodesics $p, q, r$ in $\mathscr{G}(G, S)$. Let $v$ be an $(\epsilon, R)$-transition point in $r$. Then there exists an $(\epsilon, R)$-transition point $w \in p \cup q$ such that $d(v, w)<D$.

As a special case, we obtain the following result.

Lemma 2.6 Let $\epsilon \geq \epsilon_{0}$ and $R=\mathcal{R}\left(\epsilon_{0}\right)$. For any $r>0$, there exists $D=D(r)$ with the following property. Let $p$ and $q$ be two geodesics with $p_{-}=q_{-}$and $d\left(p_{+}, q_{+}\right) \leq r$. Consider an $(\epsilon, R)$-transition point $v \in p$. Then $d(v, q) \leq D$.

Remark For convenience, it will be useful to take the congruent point $w \in q$ relative to $v \in p$ such that $d(v, w) \leq D$ in the conclusion. In particular, $d\left(p_{-}, v\right)=d\left(p_{-}, w\right)$.

\section{B Exponential growth of balls}

We now consider a type of Poincaré series associated to a subset $A \subset G$ as follows:

$$
\mathcal{P}(s, A)=\sum_{a \in A} \exp (-s \cdot d(1, a)), \quad s \geq 0 .
$$

The critical exponent $v_{A}$ of $\mathcal{P}(s, A)$ is the limit superior

$$
v_{A}=\limsup _{n \rightarrow \infty} \frac{\log |B(1, n) \cap A|}{n},
$$

which can be thought of as the exponential growth rate of $A$. Note that $v_{G}$ is the usual exponential rate $\nu_{G, S}$ of $G$ with respect to $S$. It is readily checked that $\mathcal{P}(s, A)$ is convergent for $s>v_{A}$ and divergent for $s<v_{A}$. 
Recall that a relatively hyperbolic group $G$ acts as a convergence group on its Bowditch boundary $\partial G$; see [2]. Thus every subgroup $H$ has a well-defined limit set $\Lambda(H) \subset \partial G$, which consists of the set of accumulation points of all $H$-orbits in $\partial G$. Yang proves the following result.

Lemma 2.7 [17, Lemma 4.9] Let $H$ be a subgroup in $G$ such that $\Lambda(H)$ is properly contained in $\partial G$. Then $\mathcal{P}(s, H)$ is convergent at $s=v_{G}$.

Recall that every parabolic subgroup $P \in \mathcal{P}$ fixes a unique point in $\partial G$, which coincides with the limit set $\Lambda(P)$. Lemma 2.7 then applies and the following result follows immediately.

Corollary 2.8 For every $P \in \mathcal{P}$ and $s \geq v_{G}$, we have

or, equivalently,

$$
\sum_{p \in P} \exp (-s \cdot d(1, p))<\infty
$$

$$
\sum_{n \geq 1} \exp (-s n) \cdot\left|S_{n}(P)\right|<\infty
$$

The estimate below is also important in the proof of Theorem 1.3. The lower bound holds for any group as a consequence of the submultiplicative inequality $\left|S_{n+m}\right| \leq\left|S_{n}\right|\left|S_{m}\right|$.

Lemma 2.9 [17, Theorem 1.8] Let $G$ be a relatively hyperbolic group with a finite generating set $S$. Then there exists $c>1$ such that

$$
\exp \left(n v_{G}\right) \leq\left|S_{n}\right| \leq c \cdot \exp \left(n v_{G}\right)
$$

for any $n \geq 1$.

\section{Proof of Theorem 1.3}

The proof is organized into two parts, the first of which is to decompose $S_{n}$ into the union of a sequence of $C_{R+i}$ sets; the second is to execute the calculation $\sum d(x, y)$ following the decomposition. We begin with the definition of uniform constants.

Constants 3.1 Recall that $\mathcal{R}$ is the function given by Lemma 2.2.

(1) Let $\epsilon>0$ satisfy Lemmas 2.4, 2.5 and 2.6. Assume also that $\epsilon, D_{0}>0$ are the contracting constants for $\mathbb{P}$.

(2) Let $R_{0}=\mathcal{R}(\epsilon)$.

(3) Let $D_{1}=D(0)$ given by Lemma 2.6. We also demand that $D_{1}$ satisfies Lemma 2.5. 


\section{A Defining $C_{R+i}$ sets}

Fix any number $0<\rho<\frac{1}{2}$. We consider the sphere $S_{\rho n}$ for $n \geq 1$. For simplicity, assume that $\rho n$ is an integer. We will divide $S_{n}$ into disjoint well-controlled subsets. Choose $R>\max \left\{2 R_{0}, \mathcal{R}\left(2 D_{1}\right)\right\}$. Let $C_{R}$ be the set of elements $g \in S_{n}$ such that there exists a geodesic $\gamma_{g}=[1, g]$ containing an $\left(\epsilon, R_{0}\right)$-transition point in the (closed) $R$-neighborhood of $\gamma_{g}(\rho n)$.

We consider any $g \in S_{n} \backslash C_{R}$. By definition of $C_{R}$, any geodesic $\gamma$ between 1 and $g$ will not contain an $\left(\epsilon, R_{0}\right)$-transition point in the $R$-neighborhood of $\gamma(\rho n)$. That is to say, the segment $\gamma([\rho n-R, \rho n+R])$ is contained in $N_{\epsilon}\left(X_{\gamma}\right)$ for some $X_{\gamma} \in \mathbb{P}$. We first claim the following.

Claim $X_{\gamma}$ is independent of the choice of $\gamma$.

Proof If not, we have that $\gamma, \gamma^{\prime}, X_{\gamma}$ and $X_{\gamma^{\prime}}$ satisfy the requirement as above. Note that $\gamma$ and $\gamma^{\prime}$ have the same endpoints. Let $x_{-}$and $x_{+}$be the entry and exit points of $\gamma$ in $N_{\epsilon}\left(X_{\gamma}\right)$, respectively. The points $y_{-}, y_{+} \in \gamma^{\prime}$ are similarly defined for $X_{\gamma^{\prime}}$. Thus, by Lemma 2.4, $x_{-}, x_{+}, y_{-}$and $y_{+}$are $\left(\epsilon, R_{0}\right)$-transitional points. By Lemma 2.6, it follows that

$$
d\left(x_{-}, \gamma^{\prime}\right), d\left(x_{+}, \gamma^{\prime}\right), d\left(y_{-}, \gamma\right), d\left(y_{+}, \gamma\right) \leq D_{1} .
$$

Clearly, by the remark after Lemma 2.6, we see that $N_{2 D_{1}}\left(X_{\gamma}\right) \cap N_{2 D_{1}}\left(X_{\gamma^{\prime}}\right)$ has diameter at least $2 R \geq \mathcal{R}\left(2 D_{1}\right)$. This implies that $X_{\gamma}=X_{\gamma^{\prime}}$ by the bounded intersection property of $\mathbb{P}$.

Thus, in what follows, we omit the index $\gamma$ in $X_{\gamma}$.

Let $z$ be the entry point of $\gamma$ in $N_{\epsilon}(X)$. By Lemma 2.4, $z$ is an $\left(\epsilon, R_{0}\right)$-transition point in $\gamma$. We observe that such a $z$ lies in a uniformly bounded ball.

Lemma 3.2 For any $g \in S_{n} \backslash C_{R}$, there exists a point $x \in X$ such that any geodesic $\gamma=[1, g]$ satisfies $d(x, z) \leq D_{0}+\epsilon$. In particular, the set of $z \in \gamma$ for all possible $\gamma=[1, g]$ is uniformly bounded.

Proof Let $x \in X$ be a projection point of 1 to $X$. By the contracting property of $X$, we see that $d(z, x) \leq D_{0}+\epsilon$.

We subdivide $S_{n} \backslash C_{R}$ and define a sequence of subsets as follows. For $i \geq 1$, define $C_{R+i}$ to be the set of elements $g$ in $S_{n} \backslash C_{R}$ where the point $z \in \gamma$ defined as above is nearest to 1 among all $\gamma=[1, g]$ and has an exact distance $(R+i)$ to $\gamma(\rho n)$. We require that $R+i \leq \rho n$ for obvious reasons.

We note the following fact. 
Lemma 3.3 $C_{R+i} \cap C_{R+j}=\varnothing$ for $i \neq j$.

By the above discussion, we have the following disjoint union for $S_{n}$ :

$$
\left(\bigcup_{i \geq 1} C_{R+i}\right) \cup C_{R}=S_{n} .
$$

Recall that $\mathcal{P}=\left\{P_{k} \mid 1 \leq k \leq m\right\}$ is a finite set. The following estimate is crucial in the remaining argument, saying that $C_{R}$ occupies the major part of $S_{n}$ for sufficiently large $R \gg 0$.

Lemma 3.4 For any $\varepsilon>0$, there exists $R_{1}>0$ with the following property. Let $R \geq R_{1}$ and $n \geq 1$ such that $\rho n>R$. Then

$$
\sum_{1 \leq i \leq \rho n-R}\left|C_{R+i}\right| /\left|S_{n}\right| \leq \varepsilon .
$$

Proof By definition of $C_{R+i}$, for any $g \in C_{R+i}$, there exists a geodesic $\gamma_{g}=[1, g]$ such that $\gamma_{g}([\rho n-R-i, \rho n]) \subset N_{\epsilon}(X)$ for some $X \in \mathbb{P}$. It then follows that

$$
\left|C_{R+i}\right| \leq \sum_{1 \leq k \leq m}\left|S_{\rho n-R-i}\right| \cdot|B(1, \epsilon)| \cdot\left|S_{R+i+2 \epsilon}\left(P_{k}\right)\right| \cdot|B(1, \epsilon)| \cdot\left|S_{n-\rho n}\right|,
$$

where $R+i \leq \rho n$. Note that $\left|S_{R+i+2 \epsilon}\left(P_{k}\right)\right| \leq\left|S_{R+i}\left(P_{k}\right)\right| \cdot\left|S_{2 \epsilon}\right|$ for $1 \leq k \leq m$. By Corollary 2.8 , the series

$$
\sum_{i \geq 1}\left|S_{R+i}(P)\right| \cdot \exp \left(-v_{G}(R+i)\right)<\infty
$$

is convergent for each $P \in \mathcal{P}$. The conclusion then follows as a combination of the estimate (1) of Lemma 2.9 and the convergent series (2).

\section{B Calculating the sum $\sum d(x, y)$}

We first calculate the sum $\sum d(x, y)$, where $y$ lies in $C_{R}$.

Lemma 3.5 Let $F=B\left(1,2 D_{1}\right)$ and $D_{n, R}=2\left(n-\rho n-R-D_{1}\right)>0$. Then

$$
\sum_{x \in S_{n}, y \in C_{R}} d(x, y) \geq\left|C_{R}\right| \cdot\left(\left|S_{n}\right|-|F| \cdot\left|S_{n-\rho n+R}\right|\right) \cdot D_{n, R} .
$$

Proof For any $y \in C_{R}$, there exists a geodesic $\gamma_{y}=[1, y]$ such that $\gamma_{y}$ contains an $\left(\epsilon, R_{0}\right)$-transition point $z$ in the $R$-neighborhood of $\gamma_{y}(\rho n)$. We can assume further that $z$ is nearest to $\gamma_{y}(\rho n-R)$ among all such $\gamma_{y}=[1, y]$. Then $d\left(\gamma_{y}(\rho n), z\right) \leq R$. 
We consider two sets, $A$ and $B$, of elements in $S_{n}$, separately. Let $A$ be the set of elements $x \in S_{n}$ such that $d(z,[1, x]) \leq D_{1}$ for some $[1, x]$. Thus it follows that

$$
|A| \leq|F| \cdot\left|S_{n-\rho n+R}\right| \text {. }
$$

Let $B=S_{n} \backslash A$. For any $x$ in $B$, we have $d(z,[1, x])>D_{1}$, and then $d(z,[x, y]) \leq D_{1}$ by Lemma 2.5. Observe that $d(x, z) \geq d(y, z)$. Indeed, if $d(x, z)<d(y, z)$, then $d(1, x) \leq d(x, z)+d(z, 1)<d(y, z)+d(z, 1)=d(1, y)$. This is a contradiction, since $x, y \in S_{n}$.

Let $w \in[x, y]$ such that $d(z, w) \leq D_{1}$. Note that $d(z, y) \geq n-\rho n-R$. Thus, $\min \{d(y, w), d(x, w)\} \geq n-\rho n-R-D_{1}$. Therefore, the inequality (3) holds.

We now estimate the sum $\sum d(x, y)$ where $y \in C_{R+i}$ for $i \geq 1$. The same proof as Lemma 3.5 for the case $i=0$ proves the following.

Lemma 3.6 For each $i \geq 1$ with $R+i \leq \rho n$, we have

$$
\sum_{x \in S_{n}, y \in C_{R+i}} d(x, y) \geq\left|C_{R+i}\right| \cdot\left(\left|S_{n}\right|-|F| \cdot\left|S_{n-\rho n+R+i}\right|\right) \cdot D_{n, R} .
$$

We are ready to finish the proof of Theorem 1.3. Combining all of the above inequalities in Lemmas 3.5, 3.6 and 3.4, we obtain

$$
\begin{aligned}
\sum_{x, y \in S_{n}} d(x, y) & =\sum_{i \geq 0} \sum_{x \in S_{n}, y \in C_{R+i}} d(x, y) \\
& \geq \sum_{i \geq 0}\left|C_{R+i}\right| \cdot\left(\left|S_{n}\right|-|F| \cdot\left|S_{n-\rho n+R+i}\right|\right) \cdot D_{n, R} \\
& \geq\left(\left|S_{n}\right|^{2}-\sum_{i \geq 0}|F| \cdot\left|C_{R+i}\right| \cdot\left|S_{n-\rho n+R+i}\right|\right) \cdot D_{n, R} .
\end{aligned}
$$

Therefore,

$$
\frac{1}{\left|S_{n}\right|^{2}} \sum_{x, y \in S_{n}} \frac{d(x, y)}{n} \geq 2(1-\theta(n, R))\left(1-\rho-\frac{R+D_{1}}{n}\right),
$$

where

$$
\theta(n, R)=\left(\sum_{i \geq 0}|F| \cdot\left|C_{R+i}\right| \cdot\left|S_{n-\rho n+R+i}\right|\right) /\left|S_{n}\right|^{2} .
$$

Lemma 3.7 For any $\varepsilon>0$, there exists $R_{1}>0$ with the following property. Let $R \geq R_{1}$ and $n \geq 1$ such that $\rho n \geq R+R_{1}$. Then $\theta(n, R) \leq \varepsilon$. 
Proof We first consider the sum with $i=0$. Note that $C_{R} \subset S_{n}$. By Lemma 2.9, there exists a uniform constant $\kappa>0$ such that

$$
\frac{|F| \cdot\left|C_{R}\right| \cdot\left|S_{n-\rho n+R}\right|}{\left|S_{n}\right|^{2}} \leq \frac{\kappa}{\exp \left(v_{G}(\rho n-R)\right)} .
$$

Choose $R_{1}>0$ such that $\kappa / \exp \left(v_{G} R_{1}\right) \leq \epsilon / 2$.

Now consider the sum with $i>0$. By Lemma 3.4, we may also choose $R_{1}$ such that

$$
\sum_{1 \leq i \leq \rho n-R}|F| \cdot\left|C_{R+i}\right| /\left|S_{n}\right| \leq \varepsilon / 2
$$

for $R>R_{1}$. This concludes the proof of the lemma.

Thus, for any $\varepsilon>0$, we choose $R>0$ and let $n \rightarrow \infty$ to get $E(G, S) \geq 2(1-\varepsilon)(1-\rho)$. As $\varepsilon$ and $\rho$ are arbitrary, we see $E(G, S)=2$. The proof of Theorem 1.3 is complete.

\section{Statistical hyperbolicity of direct products}

This section is devoted to the proof of Theorem 1.4. The outline is almost the same as the proof of the annulus lemma [7, Lemma 5], which is only sketched there. We provide here the details since we considered one relatively hyperbolic factor in $G \times H$, and our estimates in the proof of Theorem 1.3 are much more involved.

We consider the direct product $G \times H$ with a split generating set $S$. Let $d$ be the word metric on $G \times H$ with respect to $S$.

Denote $S_{G}=S \cap G$ and $S_{H}=S \cap H$. Then $S_{G}$ and $S_{H}$ generate $G$ and $H$, respectively. Recall that $S_{n}(X)$ denotes the part of the sphere $S_{n}$ in $X \subset G \times H$. Note that, since $S$ is split, $d(1,(g, h))=d_{S_{G}}(1, g)+d_{S_{H}}(1, h)$ for any $(g, h) \in G \times H$. Thus the sphere $S_{n}=S_{n}(G \times H)$ in $G \times H$ can be decomposed as follows:

$$
S_{n}=\bigcup_{0 \leq i \leq n} S_{i}(G) \times S_{n-i}(H) .
$$

Note that $S_{i}(G)$ coincides with the sphere of radius $i$ in the Cayley graph $\mathscr{G}\left(G, S_{G}\right)$ of $G$ with respect to $S_{G}$.

Lemma 4.1 For any fixed $0<t<1$,

$$
\frac{\left|\bigcup_{0 \leq i \leq t n} S_{i}(G) \times S_{n-i}(H)\right|}{\left|S_{n}\right|} \rightarrow 0 \text { as } n \rightarrow \infty .
$$


Proof We use $\prec$ and $\asymp$ to denote the inequality and equality, respectively, up to a computable multiplicative constant. Note that there exists $v$ with $v_{G, S_{G}}>v>v_{H, S_{H}}$ and $\left|S_{i}(H)\right| \prec \exp (v i)$ for all $i>0$. For simplicity, let $v_{G}=v_{G, S_{G}}$.

Since $G$ is relatively hyperbolic, it follows by Lemma 2.9 that $\left|S_{i}(G)\right| \asymp \exp \left(i v_{G}\right)$ for $i \geq 0$. Observe that

$$
\begin{aligned}
\frac{\left|\bigcup_{0 \leq i \leq t n} S_{i}(G) \times S_{n-i}(H)\right|}{\left|\bigcup_{t n \leq i \leq n} S_{i}(G) \times S_{n-i}(H)\right|} & \prec \frac{\sum_{0 \leq i \leq t n} \exp \left(i v_{G}\right) \exp ((n-i) v)}{\sum_{t n \leq i \leq n} \exp \left(i v_{G}\right)} \\
& \prec \frac{\exp \left(t n\left(v_{G}-v\right)\right)}{\exp \left(n\left(v_{G}-v\right)\right)\left(1-\exp \left((t n-n) v_{G}\right)\right)} \\
& \prec \frac{\exp \left((t n-n)\left(v_{G}-v\right)\right)}{1-\exp \left((t n-n) v_{G}\right)},
\end{aligned}
$$

which tends to 0 as $n \rightarrow \infty$ for any fixed $0<t<1$.

We now proceed as in Section 3 and indicate the necessary changes. Fix any number $0<\rho<\frac{1}{2}$. Assume that $1>t>\rho$.

We consider the annular-like set $A_{t n, n}:=\bigcup_{t n \leq i \leq n} S_{i}(G) \times S_{n-i}(H)$. By Lemma 4.1, we know that

$$
\left|A_{t n, n}\right| /\left|S_{n}\right| \rightarrow 1 \quad \text { as } \quad n \rightarrow \infty .
$$

Choose $R>\max \left\{2 R_{0}, \mathcal{R}\left(2 D_{1}\right)\right\}$, where $R_{0}$ and $D_{1}$ are given by Constants 3.1. We define $C_{R+i}$ sets in $A_{t n, n}$ for $i \geq 0$ as in Section 3, where $S_{n}$ is replaced by $A_{t n, n}$.

Let $C_{R}$ be the set of elements $(g, h) \in A_{t n, n}$ such that there exists a geodesic $\gamma_{g}=[1, g]$ in the Cayley graph $\mathscr{G}\left(G, S_{G}\right)$ of $G$ such that $\gamma_{g}$ contains an $\left(\epsilon, R_{0}\right)$-transition point in the (closed) $R$-neighborhood of $\gamma_{g}(\rho n)$.

We continue to subdivide $A_{t n, n} \backslash C_{R}$. For $i \geq 1$, define $C_{R+i}$ to be the set of elements $(g, h)$ in $A_{t n, n} \backslash C_{R}$ where the point $z \in \gamma$ defined in Section 3A is nearest to 1 among all $\gamma=[1, g]$ in $\mathscr{G}\left(G, S_{G}\right)$ and has an exact distance $(R+i)$ to $\gamma(\rho n)$. Therefore, $A_{t n, n}=\bigcup_{i \geq 0} C_{R+i}$ as a disjoint union.

We now prove an analogue of Lemma 3.4.

Lemma 4.2 For any $\varepsilon>0$, there exists $R_{1}>0$ with the following property. Let $R \geq R_{1}$ and $n \geq 1$ such that $\rho n>R$. Then

$$
\sum_{1 \leq i \leq \rho n-R}\left|C_{R+i}\right| /\left|A_{t n, n}\right| \leq \varepsilon
$$


Proof By definition of $C_{R+i}$, for any $(g, h) \in C_{R+i}$, there is a geodesic $\gamma_{g}=[1, g]$ such that $\gamma_{g}([\rho n-R-i, \rho n]) \subset N_{\epsilon}(X)$ for some $X \in \mathbb{P}$. It then follows that

$$
\left|C_{R+i}\right| \leq \sum_{\substack{t n \leq j \leq n \\ 1 \leq k \leq m}}\left|S_{\rho n-R-i}(G)\right| \cdot\left|S_{R+i}\left(P_{k}\right)\right| \cdot|B(1,4 \epsilon)| \cdot\left|S_{j-\rho n}(G)\right| \cdot\left|S_{n-j}(H)\right|,
$$

where $R+i \leq \rho n$ and $B(1, \epsilon)$ should be understood as the ball in the Cayley graph $\mathscr{G}\left(G, S_{G}\right)$.

By Lemma 2.9 there exists $c>1$ such that $\exp \left(l v_{G}\right) \leq\left|S_{l}(G)\right| \leq c \cdot \exp \left(l v_{G}\right)$ for any $l \geq 1$. Thus we obtain that

$$
\left|C_{R+i}\right| \leq c^{2}|B(1,2 \epsilon)| \cdot \sum_{\substack{t n \leq j \leq n \\ 1 \leq k \leq m}} \exp \left(v_{G}(j-R-i)\right) \cdot\left|S_{R+i}\left(P_{k}\right)\right| \cdot\left|S_{n-j}(H)\right| .
$$

On the other hand,

$$
\left|A_{t n, n}\right|=\sum_{t n \leq j \leq n}\left|S_{j}(G)\right| \cdot\left|S_{n-j}(H)\right| \geq \sum_{t n \leq j \leq n} \exp \left(j v_{G}\right) \cdot\left|S_{n-j}(H)\right| .
$$

In a similar manner as in the proof of Lemma 3.4, the conclusion follows as a consequence of the convergent series given by Corollary 2.8 .

Let $F=B\left(1,2 D_{1}\right)$ in $\mathscr{G}\left(G, S_{G}\right)$, and let $D_{n, R}=2\left(t n-\rho n-R-D_{1}\right)>0$. We proceed as in Lemma 3.5 to get the following:

Lemma 4.3 For each $i \geq 0$ with $R+i \leq \rho n$,

$$
\begin{aligned}
& \sum_{x \in A_{t n, n}, y \in C_{R+i}} d(x, y) \\
& \quad \geq D_{n, R} \cdot\left|C_{R+i}\right| \cdot\left(\left|A_{t n, n}\right|-\sum_{t n \leq j \leq n}|F| \cdot\left|S_{j-\rho n+R+i}(G)\right| \cdot\left|S_{n-j}(H)\right|\right) .
\end{aligned}
$$

Proof We sketch the arguments in the proof of Lemma 3.5 with necessary changes.

For any $y=\left(g_{y}, h_{y}\right) \in C_{R+i}$, there exists a geodesic $\gamma_{y}=\left[1, g_{y}\right]$ in $\mathscr{G}\left(G, S_{G}\right)$ such that $\gamma_{y}$ contains an $\left(\epsilon, R_{0}\right)$-transition point $z$ in the $(R+i)$-neighborhood of $\gamma_{y}(\rho n)$. Then $d\left(\gamma_{y}(\rho n), z\right) \leq R+i$.

Let $A$ be the set of elements $x=\left(g_{x}, h_{x}\right) \in A_{t n, n}$ such that $d_{S_{G}}\left(z,\left[1, g_{x}\right]\right) \leq D_{1}$. Thus the cardinality of $A$ is at most

$$
|F| \cdot \sum_{t n \leq j \leq n}\left|S_{j-\rho n+R+i}(G)\right| \cdot\left|S_{n-j}(H)\right| .
$$


Let $B=A_{t n, n} \backslash A$. For any $x=\left(g_{x}, h_{x}\right)$ in $B$, we have $d_{S_{G}}\left(z,\left[1, g_{x}\right]\right)>D_{1}$, and then $d_{S_{G}}\left(z,\left[g_{x}, g_{y}\right]\right) \leq D_{1}$ by Lemma 2.5 .

Let $w \in\left[g_{x}, g_{y}\right]$ such that $d(z, w) \leq D_{1}$. Then an argument as in Lemma 3.5 proves that $\min \left\{d_{S_{G}}\left(g_{y}, w\right), d_{S_{G}}\left(g_{x}, w\right)\right\} \geq t n-\rho n-R-D_{1}$. The inequality (9) then holds.

So we have the sum estimate

$\sum d(x, y)$

$x, y \in A_{t n, n}$

$$
\begin{aligned}
& =\sum_{i \geq 0} \sum_{\substack{x \in A_{t n}, n \\
y \in C_{R+i}}} d(x, y) \\
& \geq\left(\left|A_{t n, n}\right|^{2}-\sum_{i \geq 0} \sum_{t n \leq j \leq n}|F| \cdot\left|C_{R+i}\right| \cdot\left|S_{j-\rho n+R+i}(G)\right| \cdot\left|S_{n-j}(H)\right|\right) \cdot D_{n, R} .
\end{aligned}
$$

Thus,

where

$$
\begin{aligned}
\frac{1}{\left|S_{n}\right|^{2}} \sum_{x, y \in S_{n}} \frac{d(x, y)}{n} & \geq \frac{1}{\left|S_{n}\right|^{2}} \sum_{x, y \in A_{t n, n}} \frac{d(x, y)}{n} \\
& \geq 2 \frac{\left|A_{t n, n}\right|^{2}}{\left|S_{n}\right|^{2}}(1-\theta(n, R))\left(t-\rho-\frac{R+D_{1}}{n}\right),
\end{aligned}
$$

$$
\theta(n, R)=\left(\sum_{i \geq 0} \sum_{t n \leq j \leq n}|F| \cdot\left|C_{R+i}\right| \cdot\left|S_{j-\rho n+R+i}(G)\right| \cdot\left|S_{n-j}(H)\right|\right) /\left|A_{t n, n}\right|^{2} .
$$

We can prove a similar statement for $\theta(n, R)$ by the same reasoning as in Lemma 3.7.

Lemma 4.4 For any $\varepsilon>0$, there exists $R_{1}>0$ with the following property. Let $R \geq R_{1}$ and $n \geq 1$ such that $\rho n \geq R+R_{1}$. Then $\theta(n, R) \leq \varepsilon$.

Sketch of proof Recall that $A_{t n, n}=\bigcup_{t n \leq j \leq n} S_{j}(G) \times S_{n-j}(H)$. We note that $C_{R} \subset S_{n}$, and by (8), $\left|S_{n}\right| /\left|A_{t n, n}\right| \rightarrow 1$ as $n \rightarrow \infty$. For the sum with $i=0$, the following estimate suffices by Lemma 2.9:

$$
\frac{\sum_{t n \leq j \leq n}\left|S_{j-\rho n+R}(G)\right| \cdot\left|S_{n-j}(H)\right|}{\left|A_{t n, n}\right|} \prec \frac{1}{\exp \left(v_{G}(\rho n-R)\right)} \sum_{t n \leq j \leq n} \frac{1}{\exp \left(v_{G}(n-j)\right)},
$$

which tends to 0 as $(\rho n-R) \rightarrow \infty$. 
For the sum with $i \geq 1$, since $\sum_{t n \leq j \leq n}\left|S_{j-\rho n+R+i}(G)\right| \cdot\left|S_{n-j}(H)\right| \leq\left|A_{t n, n}\right|$, by Lemma 4.2, we have

$$
\sum_{1 \leq i \leq \rho n-R}\left|C_{R+i}\right| /\left|A_{t n, n}\right| \rightarrow 0 \quad \text { as } \quad R \rightarrow \infty .
$$

The proof of the lemma follows easily from the above estimates.

Finally, for any $\varepsilon>0$, we choose $R>0$ and let $n \rightarrow \infty$ to get $E(G, S) \geq 2(1-\varepsilon)(t-\rho)$. As $\varepsilon, t, \rho$ are arbitrary, we then obtain that $E(G, S)=2$. This completes the proof of Theorem 1.4.

\section{References}

[1] B H Bowditch, Geometrical finiteness for hyperbolic groups, J. Funct. Anal. 113 (1993) 245-317 MR

[2] B H Bowditch, Relatively hyperbolic groups, Internat. J. Algebra Comput. 22 (2012) MR

[3] M Coornaert, Mesures de Patterson-Sullivan sur le bord d'un espace hyperbolique au sens de Gromov, Pacific J. Math. 159 (1993) 241-270 MR

[4] F Dahmani, Combination of convergence groups, Geom. Topol. 7 (2003) 933-963 MR

[5] S Dowdall, M Duchin, H Masur, Statistical hyperbolicity in Teichmüller space, Geom. Funct. Anal. 24 (2014) 748-795 MR

[6] C Druţu, M Sapir, Tree-graded spaces and asymptotic cones of groups, Topology 44 (2005) 959-1058 MR

[7] M Duchin, S Lelièvre, C Mooney, Statistical hyperbolicity in groups, Algebr. Geom. Topol. 12 (2012) 1-18 MR

[8] M Duchin, C Mooney, Fine asymptotic geometry in the Heisenberg group, Indiana Univ. Math. J. 63 (2014) 885-916 MR

[9] V Gerasimov, Expansive convergence groups are relatively hyperbolic, Geom. Funct. Anal. 19 (2009) 137-169 MR

[10] V Gerasimov, L Potyagailo, Non-finitely generated relatively hyperbolic groups and Floyd quasiconvexity, Groups Geom. Dyn. 9 (2015) 369-434 MR

[11] V Gerasimov, L Potyagailo, Quasiconvexity in relatively hyperbolic groups, J. Reine Angew. Math. 710 (2016) 95-135 MR

[12] M Gromov, Hyperbolic groups, from: "Essays in group theory", (S M Gersten, editor), Math. Sci. Res. Inst. Publ. 8, Springer, New York (1987) 75-263 MR

[13] G C Hruska, Relative hyperbolicity and relative quasiconvexity for countable groups, Algebr. Geom. Topol. 10 (2010) 1807-1856 MR 
[14] G C Hruska, B Kleiner, Hadamard spaces with isolated flats, Geom. Topol. 9 (2005) 1501-1538 MR

[15] J Osborne, Statistical Hyperbolicity of Relatively Hyperbolic Groups, PhD thesis, The University of Wisconsin, Milwaukee (2014) MR Available at http:// search.proquest . com/docview/1640775426

[16] D V Osin, Relatively hyperbolic groups: intrinsic geometry, algebraic properties, and algorithmic problems, Mem. Amer. Math. Soc. 843, Providence, RI (2006) MR

[17] W-y Yang, Patterson-Sullivan measures and growth of relatively hyperbolic groups, preprint (2013) arXiv

[18] W-y Yang, Growth tightness for groups with contracting elements, Math. Proc. Cambridge Philos. Soc. 157 (2014) 297-319 MR

University of Wisconsin, Mathematics and Physics Department Kenosha, WI 53144, United States

Beijing International Center for Mathematical Research \& School of Mathematical Sciences Peking University, Beijing, 100871, China dr.j.a.osborne@gmail.com, wyang@math.pku.edu.cn http://bicmr.pku.edu.cn/ wyang

Received: 30 April $2015 \quad$ Revised: 8 September 2015 